\title{
Impact of COVID-19 in early breast cancer management: a summary of the current evidence
}

Francisco Pimentel Cavalcante ${ }^{1,2}$, Edson Abdala ${ }^{3}$, Leonardo Weissmann ${ }^{4,5,6}$, Carlos Eduardo dos Santos Ferreira ${ }^{7,8}$, Gilberto Amorim ${ }^{9,10}$, Gustavo Aguiar Campana ${ }^{8,11}$, Vilmar Marques de Oliveira ${ }^{2,12,13}$, Gisah Guilgen ${ }^{10,14,15}$, Luciana Landeiro ${ }^{16}$, João Renato Rebello Pinho $^{7,17}$, Álvaro Pulchinelli $\mathrm{Jr}^{8,18,19}$, Heber Ribeiro ${ }^{20,21}$, Rafael Souza ${ }^{22}$, and Daniela Dornelles Rosa ${ }^{10,23,24}$

${ }^{1}$ Fortaleza General Hospital, Breast Surgery

${ }^{2}$ Brazilian Society of Mastology

${ }^{3}$ USP, School of Medicine, Department of Infectious and Parasitic Diseases

${ }^{4}$ Emílio Ribas Infectious Disease Institute

${ }^{5}$ Unaerp Medicina, Guarujá

${ }^{6}$ Brazilian Society of Infectology

${ }^{7}$ Albert Einstein Hospital

${ }^{8}$ Brazilian Society of Clinical Pathology/Laboratory Medicine

${ }^{9}$ Oncologia D'Or

${ }^{10}$ GBECAM

${ }^{11}$ DASA

${ }^{12}$ Santa Casa de São Paulo Hospital

${ }^{13}$ Santa Casa de São Paulo School of Medical Sciences

${ }^{14}$ Curitiba Cancer and Transplant Institute

${ }^{15}$ Nossa Senhora das Graças Hospital

${ }^{16}$ Núcleo de Oncologia da Bahia (NOB) - Oncoclínicas Group

${ }^{17}$ Hospital das Clínicas, School of Medicine, University of São Paulo, Medical Research

${ }^{18}$ UNIFESP, School of Medicine (EPM)

${ }^{19}$ Fleury Group

${ }^{20}$ Brazilian Society of Oncology Surgery

${ }^{21}$ AC Camargo Cancer Center

${ }^{22}$ Cancer Treatment Institute (ITC)

${ }^{23}$ Moinhos de Vento Hospital

${ }^{24}$ Federal University of Health Sciences of Porto Alegre (UFCSPA)

June 21, 2021

\footnotetext{
Abstract

Purpose: An Expert Panel on Breast Cancer and COVID-19 was convened to address the impact of the COVID-19 pandemic for early breast cancer management.

Methods: In order to ensure the most clinically relevant information was addressed, essential information was drawn from several of the latest national and international guidelines and another technical document. The Expert Panel met in five virtual
} 
closed sessions from November 2020 to May 2021 to consult on the relevant data from evidence-based results. The data gathered were discussed on an online platform (Within3 $\AA$ ).

Results: This paper reports the Expert Panel's highlights of these meetings' discussions. In addition, it provides practical recommendations covering topics regarding diagnosis, treatment, and management of breast cancer patients in clinical settings routinely encountered by HCPs amid the COVID-19 pandemic.

Conclusions: It was provided guidance on several topics regarding eBC management amid the COVID-19 pandemics to inform safer care practices. 


\section{Introduction}

In 2020, the predicted number of new breast cancer cases was 2.3 million worldwide, with an estimated age-standardized rate (ASR) incidence of 47.8 per 100,000 person-years and ASR mortality of 13.6 per 100,000 person-year with 684,996 deaths predicted ${ }^{1}$. The COVID-19 pandemic has challenged the medical community on many fronts, having a significant impact on access to cancer diagnosis and treatment ${ }^{2}$. The fear of becoming infected while using healthcare facilities, fueled by the rising number of infected individuals seeking medical care, is one of the main factors delaying cancer diagnosis and treatment ${ }^{3-5}$. A significant decrease in cancer diagnoses has been observed during the COVID-19 pandemic, with the most marked decline seen in breast cancer care $(51.8 \%)^{6}$.

Surgery remains the main curative treatment for breast cancer ${ }^{7}$. However, due to the COVID-19 pandemic, breast cancer teams have been forced to review triage for surgical procedures in a bid to optimize clinical resource usage. This move has entailed assessing risks and deciding which surgery cases should be postponed ${ }^{8}$, such as elective surgeries ${ }^{9}$ and taking preventive measures for potentially infected non-deferrable surgery candidates ${ }^{10,11}$. Brazil is currently facing one of the worst moments of the pandemic, with almost 500,000 deaths registered ${ }^{12}$. The purpose of this review is to provide an evidence-based update of the management of early breast cancer during COVID-19 outbreak, with a particular emphasis on avoiding risks to both patients and healthcare professionals (HCP).

\section{Methods}

With the aim of pooling information on the host of clinical scenarios in which early breast cancer patients may present during the COVID-19 pandemic, a group of specialists in Brazil were invited to join an expert panel. In order to ensure the most clinically-relevant information was addressed, essential information was drawn from several of the latest national and international guidelines and from other technical documents $4,9,10,13-31$. The data gathered were discussed on an online platform (Within3 @ ) covering topics regarding diagnosis, treatment, and management of breast cancer patients in clinical settings routinely encountered by HCPs amid the COVID-19 pandemic.

Fourteen recognized experts joined an online expert panel and worked collaboratively in five virtual closed sessions from November 18th to May $25^{\text {th }}$, 2021, in five virtual closed sessions. A three-step process was conducted: [1] Prework, in which all relevant material was shared, and notes on crucial aspects acknowledged; [2] Steering Committee meeting, where participants discussed and shared clinical expertise, drafting recommendations; [3] Meeting convening all experts, in which a comprehensive review of all evidence provided was performed online, and resultant recommendations discussed and refined.

\section{Clinical Presentation of Breast Cancer}

Breast cancer $(\mathrm{BC})$ is a heterogeneous disease with different subtypes. Most patients with breast cancer are asymptomatic (findings from screening mammography) while others may present with a palpable lump at diagnosis. Early breast cancer (eBC; stages I and II) represents more than $75 \%$ of cases in most parts of the world ${ }^{32}$. The management of eBC is well-defined according to international protocols ${ }^{13,14,33}$. HER2-positive and triple negative (TN) BC are biologically more aggressive tumors whereas luminal cancers (which express hormone receptors) are more indolent ${ }^{34}$. Based on the Ki-67 proliferation index, the St Gallen Consensus defines two luminal subtypes: luminal A (better prognosis) and luminal B (more aggressive disease) ${ }^{34}$. Surgery is the mainstay treatment for $\mathrm{eBC}$, and the procedure may be performed upfront or after neoadjuvant therapy (chemotherapy or endocrine therapy). As a rule, HER2-positive, luminal B and TN patients are priority categories for urgent breast cancer therapy ${ }^{33}$. 


\section{Pathophysiology}

Cancer patients have dysregulated immunity with depleted immune cells, such as CD8+ Tcells, CD4+ Tcells, NK cells and others ${ }^{35}$. COVID-19 infection in cancer patients significantly increases inflammatory factors and cytokines (high-sensitivity C-reactive protein, procalcitonin, IL-2, IL-6, IL-8), possibly explaining the poorer prognosis in individuals with cancer relative to those without cancer ${ }^{36}$. SARS-CoV-2 can enter the cell by mediating spike proteins using the angiotensin-converting enzyme 2 (ACE2) receptor via plasma membrane fusion or endosomes ${ }^{37}$. SARS-CoV-2 stimulates the innate immune system and antigen-specific responses of $\mathrm{B}$ and $\mathrm{T}$ cells through a mechanism similar to that seen for the influenza virus ${ }^{38}$. The development of virusneutralizing antibodies is essential for protection against viral infections, and clinical studies of SARS-CoV-2 vaccines have been pursuing this therapeutic target ${ }^{39}$.

\section{Management}

\section{Assessment and Diagnosis}

In the context of the COVID-19 pandemic, the management of patients with eBC has become more complex, as patients can present in any of three clinical situations: asymptomatic patients with no known exposure to SARS-CoV-2, asymptomatic patients exposed to SARS-CoV-2, and symptomatic patients with suspected SARS-CoV-2 infection (Table 1) ${ }^{40}$.

The diagnosis of SARS-CoV-2 can be established based on the RT-PCR test for symptomatic or asymptomatic patients exposed within 5-10 days to SARS-CoV-2 infected patients ${ }^{41,42}$. Serological tests, such as ELISA IgM, can be employed for screening symptomatic patients after day 10 of symptoms as an alternative method to RT-PCR for COVID-19 diagnosis (gold standard) ${ }^{42}$. However, the use of serological tests alone is not recommended because they are less sensitive before 10 days of symptom onset and given the possibility of false positives ${ }^{23,24}$.

\section{Hosted file}

Table 1- Early breast cancer patients with surgery planned and SARS-CoV-2 clinical status.jpg available at https://authorea.com/users/420763/articles/527023-impact-of-covid-19-in-earlybreast-cancer-management-a-summary-of-the-current-evidence

Another practical approach is to assess eBC management in those cases with SARS-CoV-2 test results available (positive or negative) and a more controversial clinical scenario (Table 2).

\section{Consider using Point of Care (POC) antigen tests if PCR unavailable? In what situations?}

Antigen-detection for the diagnosis of SARS-CoV2 using point-of-care tests (POC) provides a workable solution that could enable patients to self-isolate earlier and reduce the spread of infection ${ }^{17}$, representing an option which is accessible to most outbreak areas compared with standard nucleic acid amplification tests (NAATs), such as RT-PCR assays ${ }^{18}$. However, the trade-off is a loss of sensitivity compared to NAATs, particularly among asymptomatic patients ${ }^{44}$. These tests should be carried out by trained professionals.

The POC antigen test is a viable when RT-PCR is unavailable in the following scenarios ${ }^{18}$ : 
* $\quad$ Patients presenting with 5-7-day onset of symptoms;

* Positive results need confirmation by RT-PCR assays (ideally);

* Outbreak areas and remote settings, where POC testing constitute an alternative to RT-PCR.

\section{Consider using Point of Care (POC) antibody tests if PCR was unavailable? In what situations?}

Serology tests have limited application diagnosis-wise, particularly in the acute phase ${ }^{45}$, as most patients will develop an antibody response within 1 to 3 weeks after infection ${ }^{19}$. Crucial windows of opportunity for clinical intervention and isolation measures may have already been missed ${ }^{19}$.

There is also a possibility of cross-reaction with other pathogens, such as other human coronaviruses, increasing the odds for false positives ${ }^{45}$. There was no consensus among the experts regarding the clinical utility of POC antibody tests. Some authors agreed this technology could be considered in some situations, despite its limitations in order to ${ }^{19}$ :

* determine the extent of infection in patients not diagnosed using RT-PCR

* determine infection fatality rate

* $\quad$ support the development of vaccines

\section{Hosted file}

Table 2- SARS-CoV-2 test results in specific eBC clinical scenarios.jpg available at https : //authorea.com/users/420763/articles/527023-impact-of-covid-19-in-early-breastcancer-management-a-summary-of-the-current-evidence

\section{Treatment}

\section{Neoadjuvant therapy to allow delay of surgery}

The clinical management guidelines for breast cancer were recently updated in the COVID-19 era. Clinical cases eligible for neoadjuvant treatment are ${ }^{9,24}$ :

* Triple-Negative Breast Cancer (TNBC), HER2-positive and luminal B tumors $>/=2 \mathrm{~cm}$ and $/$ or with positive axilla ([?]N1).

* Luminal A tumors stages T1-T2 and N0-N1 (neoadjuvant endocrine therapy [NET] may be recommended, especially in postmenopausal patients).

* Inflammatory and locally advanced breast cancer (NET or neoadjuvant chemotherapy [NCT]).

* $\quad$ Any type - to complete NCT that has already been initiated.

Specifically, for ER-positive and HER2-negative patients, both ESMO and ACS have stated that NET is an option to enable deferral of surgery by 6 to 12 months in clinical stage I or II breast cancers according to menopausal status ${ }^{9,24}$.

Although constraints are often present in terms of resources, workforce, and hospital bed availability in the COVID-19 pandemic, causing a delay in procedures, both NET and NCT appear to be safe choices to postpone surgery in non-urgent indications of ER-positive early-stage breast cancer, also potentially contributing to a reduction in outpatient visits. 


\section{Managing axilla after neoadjuvant systemic therapy}

According to the panel, if the sentinel lymph node (SN) is negative at the time of surgery, axillary dissection $(\mathrm{AD})$ is not recommended, even in previously positive axilla. If the $\mathrm{SN}$ is positive, however, then course of action should be discussed on a case-by-case basis, especially after NET ${ }^{48}$.

There has been increasing interest in omitting AD after NCT in past years, even in those with residual disease on sentinel lymph node biopsy (SNB). A recent American study ${ }^{49}$ demonstrated that the use of isolated positive SN after NCT has an upward trend after the publication of the results of ACOSOG Z0011

50. In fact, the Z0011 study demonstrated excellent local and locoregional control with isolated SLNB but excluded patients who underwent neoadjuvant systemic treatment (NCT or NET) ${ }^{50}$. In women undergoing NCT, residual axillary disease can be associated with resistance, and there is no data on cancer safety when omitting axillary dissection at this time. A retrospective review evaluated residual disease burden in positive SN after NCT and demonstrated an additional high disease burden, regardless of whether it was micrometastasis $(59 \%)$ or macrometastases $(63 \%)$, possibly an indication for $\mathrm{AD}^{51}$. Another analysis showed that the likelihood of non-SLN-centered metastasis at axillary lymph node dissection (ALND) was high across all tumor subtypes ${ }^{52}$. The core point is whether $\mathrm{AD}$ would play a role in residual lymph node disease cases or whether axillary radiation therapy could replace surgery in such cases. For instance, a retrospective study using data from the National Cancer Database (NCDB), with 1617 women with N1 disease after $\mathrm{NACT}$, compared patients who received $\mathrm{AD}$ associated with nodal radiotherapy with those who received only SNB and radiotherapy, similar to the design of an ongoing randomized study of ALLIANCE group (A11202) ${ }^{53}$ showing increased survival in women undergoing AD ${ }^{54}$. However, in an exploratory analysis, the authors found that SN was comparable to AD in luminal tumors with single metastases. The panel recommends caution in omitting AD in such cases.

On the other hand, after NET, pathologic complete response (pCR) is generally not expected following systemic treatment ${ }^{55}$. The question is whether these patients match the ACOSOG Z0011 study profile or otherwise. The data in this scenario is limited. A study using the NCDB and Dana-Farber/Brigham and Women's Cancer Center database evaluated tumor burden after NET and the type of axillary surgery performed (SNB or $\mathrm{AD}$ ): more than $90 \%$ of patients who had $\mathrm{cN0}$ axilla at initial presentation, in both cohorts, they had $<3$ positive lymph nodes in the final pathology, with no difference in overall survival regardless of the type of axillary surgery ${ }^{48}$. In another study, using the NCDB, for stages 2 and 3 , SNB use after NET was similar to that for upfront surgery and, among those with pN1 disease, the NET patients were less likely to undergo $\mathrm{AD}^{56}$. In this scenario, the panel recommended a case-by-case assessment, with the possibility of omitting axillary dissection, especially in initially clinically negative axilla.

\section{Breast conservative surgery and risk of infection by COVID-19}

BCS is associated with lower rates of hospital stay and visits after surgery and hospitalization than mastectomy ${ }^{57}$ : a study with patients undergoing nipple-sparing mastectomy (NSM) had total complication rates of $47 \%$ and reoperations around $9 \%{ }^{58}$. Regarding the use of oncoplastic surgery, complication rates also tend to be higher than in BCS. In a study using the American College of Surgeons National Surgical Quality Improvement Program (ACS NSQIP) database, complications within 30 days were greater in patients undergoing oncoplastic surgery compared to BCS $(3,8 \%$ vs. $2.6 \%$; p < 0.001$){ }^{59}$. Another prospective cohort (TeaM Study) identified a reoperation rate of $2.8 \%{ }^{60}$. In a survey conducted during the pandemic among mastologists from the Brazilian Society of Mastology (SBM), $75 \%$ of surgeons would recommend partial reconstruction after BCS; however, $54 \%$ of those would contraindicate mammoplasty techniques during the pandemic period ${ }^{61}$. The panel recommends caution in recommending major surgery during the pandemic. 
Although there are still limited data on this subject, it is possible to infer that the risk of contamination for less invasive surgeries, such as BCS, is low because risks of procedure complications and surgery times are lower. In addition, all precautions mentioned previously should also be taken for this surgical procedure.

\section{Elective surgeries that cannot be delayed}

Elective surgeries, by definition, can be postponed for up to 8 weeks. There are a few elective situations that are considered essential and require planned or immediate medical assistance surgery-wise. Emergency or urgent surgeries are those that, if not performed might compromise patient survivorship. Examples of this type of surgery are revision of an ischemic mastectomy flap, surgical evacuation of breast hematoma, drainage of breast abscess and revascularization of an autologous tissue flap ${ }^{9}$.

\section{Bilateral mastectomy}

Regarding patients with contralateral prophylactic mastectomy in unilateral breast cancer indication, although there is still limited data on this subject, historically these cases have a longer hospital stay compared to breast-conserving surgery or unilateral mastectomy, and also have more post-surgery visits and higher rates of hospitalization ${ }^{57}$. This potential increase in patient exposure could lead to a greater risk of infection by COVID-19 ${ }^{25}$. The expert panel suggested that a contralateral prophylactic mastectomy is not recommended during this period and conservative breast surgery, or even unilateral mastectomy should be carried out instead. The panel recommended that immediate breast reconstruction should be evaluated on a case-by-case basis, according to the local conditions or resource availability due to the pandemics.

\section{COVID-19 vaccines and breast cancer}

According to the panel, breast cancer patients should receive the COVID-19 vaccine as soon as it becomes available, since benefits are likely to outweigh the risks of adverse effects from SARS-CoV-2 vaccination ${ }^{62}$. The National Comprehensive Cancer Network (NCCN) and the European Society for Medical Oncology recently reinforced this position ${ }^{26,27}$. It is essential to point out that there is limited clinical data supporting COVID-19 vaccination in cancer patients ${ }^{63}$. A multicenter, observational, prospective study has shown that SARS-CoV-2 specific IgG antibody response does not differ in cancer patients and HCP ${ }^{64}$. It is uncertain, however, whether long-term immunization can be achieved in the oncologic population ${ }^{63}$. In the same vein, data from influenza vaccinations indicates the development of immune protective response in cancer patients and, although, potentially, not the same level as compared to the general population, it is generally safe 28,65-67. Again, there are long-term uncertainties, and the protection may vary depending on antineoplastic therapies, administration timing, disease stage, and comorbidities ${ }^{68}$.

It is important to note that patients who received monoclonal antibodies or convalescent plasma as part of COVID-19 treatment should defer vaccination for at least 90 days as stated by CDC recommendations 29. After the final dose is received, an individual is considered fully vaccinated after a minimum of two weeks ${ }^{30}$. If the patient is asymptomatic and has not been in close contact with someone with SARS-CoV-2 infection in the prior 14 days, the panel deemed it safe to conduct a surgical procedure. The expert panel recommendation for vaccination against COVID-19 for $\mathrm{eBC}$ is presented in Figure 1. Table 3 summarizes the main vaccines approved worldwide as of April 14th, 2021.

Recently, an unexpectedly high incidence of axillary adenopathy findings after Moderna and Pfizer-BioNTech COVID-19 vaccines occurred ${ }^{69}$. For patients receiving the Moderna vaccine, a solicited adverse event was reported in $11.6 \%$ vs $5.0 \%$ for placebo following dose 1 , and $16.0 \%$ vs $4.3 \%$ for placebo following dose $2^{70}$. Adenopathy occurred in the arm and neck 2-4 days after vaccination with a median duration of 1-2 days ${ }^{69}$. For those receiving the Pfizer-BioNTech vaccine, resultant lymphadenopathy lasted for a mean of 10 days. 
However, in the Pfizer-BioNTech study, adenopathy was only reported as an unsolicited adverse event ${ }^{69}$. A single institution report found similar findings and the authors are considering "MRI-detected isolated unilateral lymphadenopathy ipsilateral to the vaccination arm to be most likely COVID-19 vaccine-related if within four weeks of either dose" 71 .

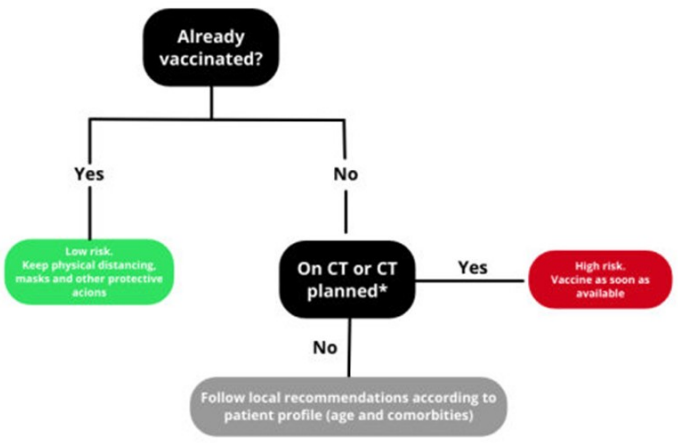

Figure 1: Vaccination against COVID-19 for eBC. CT: chemotherapy; * Planned within 8 weeks

\section{What is the role of post-vaccine antibody quantification tests in $\mathrm{eBC}$ patients?}

The current evidence supports that seroconversion rates among patients with cancer are similar to those without the disease, particularly in solid tumors like breast cancer ${ }^{72}$. Vaccine-wise, serological tests can often be misinterpreted as they might not distinguish between past infection and post-vaccination immunological response ${ }^{23}$. Furthermore, serologic testing does not evaluate cellular immune response. When performed against nucleocapsid protein, these tests will not detect immune responses resulting from vaccination, and are not suitable for vaccine decision-making ${ }^{29}$. Most experts do not see a clinical application for these tests.

\section{Hosted file}

Table 3-\selectlanguage\{ngerman\} COVID-19 vaccine candidates approved.jpg available at https : //authorea.com/users/420763/articles/527023-impact-of-covid-19-in-early-breastcancer-management-a-summary-of-the-current-evidence

\section{Conclusion}

We have provided guidance on several topics regarding eBC management amid the COVID-19 pandemic to inform safer care practices for both patients and HCPs. 


\section{Funding}

Roche Diagnostics supported this work.

\section{Disclosure statement}

Dr. Cavalcante reports compensation for speaker's bureau and honoraria from Gencell Pharma, Libbs, Pfizer and Roche. Also, Dr. Cavalcante reported serving on the advisory board for MSD, Roche, and Pfizer. Dr. Amorim participated in advisory boards, received honoraria, and was invited by Roche to medical conferences. Dr. Ribeiro reports compensation for speaker's bureau and honoraria from Roche. Dra. Rosa reported serving on consulting and advisory board for Amgen, AstraZeneca, Dr. Reddy, Eisai, GSK, Libbs, Lilly, Novartis, Pfizer, Roche, Sanofi, Teva, United Medical, and Zodiac. No other disclosures were reported.

\section{Acknowledgements}

All authors contributed to writing the article and were approved to submit it for publication. In addition, the authors thank Dr. Alexandre Ferreira Oliveira, Dr. Reitan Ribeiro, and Dr. Ruffo Freitas-Junior for their review and inputs. CoreBox Medical Communications provided medical writing assistance.

\section{References}

1. Sung H, Ferlay J, Siegel RL, et al. Global cancer statistics 2020: GLOBOCAN estimates of incidence and mortality worldwide for 36 cancers in 185 countries. CA: a cancer journal for clinicians. 2021.

2. Siegel RL, Miller KD, Fuchs HE, Jemal A. Cancer Statistics, 2021. CA: a Cancer Journal for Clinicians. 2021;71(1):7-33.

3. Aguiar S, Baiocchi G, Duprat JP, et al. Value of preoperative testing for SARS-CoV-2 for elective surgeries in a cancer center during the peak of pandemic in Brazil. Journal of Surgical Oncology. 2020;122(7):1293-1295. doi:https://doi.org/10.1002/jso.26146

4. Cavalcante FP, Novita GG, Millen EC, et al. Management of early breast cancer during the COVID-19 pandemic in Brazil. Breast Cancer Res Treat. 2020;184(2):637-647. doi:10.1007/s10549-02005877-y

5. Papautsky EL, Hamlish T. Patient-reported treatment delays in breast cancer care during the COVID-19 pandemic. Breast Cancer Res Treat. 2020;184(1):249-254. doi:10.1007/s10549-020-05828-7

6. Kaufman HW, Chen Z, Niles J, Fesko Y. Changes in the Number of US Patients With Newly Identified Cancer Before and During the Coronavirus Disease 2019 (COVID-19) Pandemic. JAMA Network Open. 2020;3(8):e2017267-e2017267. doi:10.1001/jamanetworkopen.2020.17267

7. Czajka ML, Pfeifer C. Breast Cancer Surgery. In: StatPearls. Treasure Island (FL): StatPearls Publishing; 2020. http://www.ncbi.nlm.nih.gov/books/NBK553076/. Accessed January 13, 2021. 
8. Bartlett DL, Howe JR, Chang G, et al. Management of Cancer Surgery Cases During the COVID-19 Pandemic: Considerations. Ann Surg Oncol. 2020;27(6):1717-1720. doi:10.1245/s10434-020-08461-2

9. American College of Surgeons. COVID-19 Guidelines for Triage of Breast Cancer Patients.https://www.facs.org/covid-19/clinical-guidance/elective-case/breast-cancer. Accessed January 15, 2021.

10. Pryor A. SAGES and EAES recommendations regarding surgical response to COVID-19 crisis.https://www.sages.org/recommendations-surgical-response-covid-19. Published March 30, 2020. Accessed January 15, 2021.

11. Hwang ES, Balch CM, Balch GC, et al. Surgical Oncologists and the COVID-19 Pandemic: Guiding Cancer Patients Effectively through Turbulence and Change. Ann Surg Oncol. 2020;27(8):26002613. doi:10.1245/s10434-020-08673-6

12. Ministério da Saúde. Brasil. Coronavírus Brasil. https://covid.saude.gov.br/. Published 2021. Accessed June 7, 2021.

13. National Comprehensive Cancer Network (NCCN). Breast Cancer - Version 1.2021 - January 15, 2021. https://www.nccn.org/professionals/physician_gls/pdf/breast.pdf. Accessed January 15, 2021.

14. Cardoso F, Kyriakides S, Ohno S, et al. Early breast cancer: ESMO Clinical Practice Guidelines for diagnosis, treatment and follow-up+. Annals of Oncology. 2019;30(8):1194-1220. doi:10.1093/annonc/mdz173

15.

National Institute for Health and Care Excellence (NICE). COVID-19 rapid guideline: arranging planned care in hospitals and diagnostic services: NICE guideline [NG179].https://www.nice.org.uk/guidance/ng179. Accessed April 14, 2021.

16. Ribeiro R, Wainstein AJA, de Castro Ribeiro HS, Pinheiro RN, Oliveira AF. Perioperative Cancer Care in the Context of Limited Resources during the COVID-19 Pandemic: Brazilian Society of Surgical Oncology Recommendations. Ann Surg Oncol. September 2020. doi:10.1245/s10434-020-09098-x

17. Dinnes J, Deeks JJ, Adriano A, et al. Rapid, point-of-care antigen and molecularbased tests for diagnosis of SARS-CoV-2 infection. Cochrane Database of Systematic Reviews. 2020;(8). doi:10.1002/14651858.CD013705

18. World Health Organization (WHO). Antigen-detection in the diagnosis of SARS-CoV-2 infection using rapid immunoassays. https://www.who.int/publications/i/item/antigen-detection-in-the-diagnosis-ofsars-cov-2infection-using-rapid-immunoassays. Accessed April 16, 2021.

19. Centers for Disease Control and Prevention (CDC). Using Antibody Tests for COVID-19.https://www.cdc.gov/coronavirus/2019-ncov/lab/resources/antibody-tests.html. Accessed April $14,2021$.

20. World Health Organization (WHO). Advice on the use of point-of-care immunodiagnostic tests for COVID-19: scientific brief. https://www.who.int/news-room/commentaries/detail/advice-on-the-use-ofpoint-of-care-immunodiagnostic-tests-for-covid-19. Published 2020. Accessed April 16, 2021.

21. Centers for Disease Control and Prevention (CDC). Sequence for putting on personal protective equipment (PPE). https://www.cdc.gov/hai/pdfs/ppe/ppe-sequence.pdf. Accessed January 15, 2021.

22. Centers for Disease Control and Prevention (CDC). Clinical Questions about COVID-19: Questions and Answers.https://www.cdc.gov/coronavirus/2019-ncov/hcp/faq.html. Accessed April 14, 2021.

23. Centers for Disease Control and Prevention (CDC). Interim Guidelines for COVID-19 Antibody Testing.https://www.cdc.gov/coronavirus/2019-ncov/lab/resources/antibody-tests-guidelines.html. Accessed April 14, 2021. 
24. European Society for Medical Oncology (ESMO). ESMO Management and Treatment Adapted Recommendations in the COVID-19 Era: Breast Cancer.https://www.esmo.org/guidelines/cancer-patientmanagement-during-the-covid-19-pandemic/breast-cancer-in-the-covid-19-era. Accessed January 15, 2021.

25. Centers for Disease Control and Prevention (CDC). Interim U.S. Guidance for Risk Assessment and Work Restrictions for Healthcare Personnel with Potential Exposure to SARS-CoV2.https://www.cdc.gov/coronavirus/2019-ncov/hcp/guidance-risk-assesment-hcp.html. Accessed April 14, 2021.

26. National Comprehensive Cancer Network (NCCN). Recommendations of the NCCN COVID-19 Vaccination Advisory Committee. https://www.nccn.org/covid-19. Accessed April 16, 2021.

27. European Society for Medical Oncology (ESMO). ESMO Statements for vaccination against COVID19 in patients with cancer.https://www.esmo.org/covid-19-and-cancer/covid-19-vaccination. Accessed April $16,2021$.

28. Bitterman R, Eliakim-Raz N, Vinograd I, Zalmanovici Trestioreanu A, Leibovici L, Paul M. Influenza vaccines in immunosuppressed adults with cancer. Cochrane Database Syst Rev. 2018;2:CD008983. doi:10.1002/14651858.CD008983.pub3

29. Centers for Disease Control and Prevention (CDC). Interim Clinical Considerations for Use of COVID-19 Vaccines Currently Authorized in the United States.https://www.cdc.gov/vaccines/covid19/info-by-product/clinical-considerations.html. Accessed April 14, 2021.

30. Centers for Disease Control and Prevention (CDC). Updated Healthcare Infection Prevention and Control Recommendations in Response to COVID-19 Vaccination.https://www.cdc.gov/coronavirus/2019ncov/hcp/infection-control-after-vaccination.html. Accessed April 14, 2021.

31. Centers for Disease Control and Prevention (CDC). Local Reactions, Systemic Reactions, Adverse Events, and Serious Adverse Events: Moderna COVID-19 Vaccine.https://www.cdc.gov/vaccines/covid19/info-by-product/moderna/reactogenicity.html. Accessed April 14, 2021.

32. Simon SD, Bines J, Werutsky G, et al. Characteristics and prognosis of stage I-III breast cancer subtypes in Brazil: The AMAZONA retrospective cohort study. Breast. 2019;44:113-119. doi:10.1016/j.breast.2019.01.008

33. Dietz JR, Moran MS, Isakoff SJ, et al. Recommendations for prioritization, treatment, and triage of breast cancer patients during the COVID-19 pandemic. the COVID-19 pandemic breast cancer consortium. Breast Cancer Res Treat. April 2020:1-11. doi:10.1007/s10549-020-05644-z

34. Coates AS, Winer EP, Goldhirsch A, et al. Tailoring therapies-improving the management of early breast cancer: St Gallen International Expert Consensus on the Primary Therapy of Early Breast Cancer 2015. Annals of Oncology. 2015;26(8):1533-1546. doi:10.1093/annonc/mdv221

35. Marcus A, Gowen BG, Thompson TW, et al. Recognition of tumors by the innate immune system and natural killer cells. Advances in immunology. 2014;122:91-128.

36. Cai G, Gao Y, Zeng S, et al. Immunological alternation in COVID-19 patients with cancer and its implications on mortality. Oncoimmunology. 2021;10(1):1854424. doi:10.1080/2162402X.2020.1854424

37. Hoffmann M, Kleine-Weber H, Schroeder S, et al. SARS-CoV-2 Cell Entry Depends on ACE2 and TMPRSS2 and Is Blocked by a Clinically Proven Protease Inhibitor. Cell. 2020;181(2):271-280.e8. doi:10.1016/j.cell.2020.02.052

38. Thevarajan I, Nguyen THO, Koutsakos M, et al. Breadth of concomitant immune responses prior to patient recovery: a case report of non-severe COVID-19. Nature Medicine. 2020;26(4):453-455. doi:10.1038/s41591-020-0819-2 
39.

Han HJ, Nwagwu C, Anyim O, Ekweremadu C, Kim S. COVID-19 and cancer: From basic mechanisms to vaccine development using nanotechnology. Int Immunopharmacol. 2021;90:107247. doi:10.1016/j.intimp.2020.107247

40. Ferreira CE, Bonvehi PE, Torre JCG de la, Saenz-Flor KV, Condino-Neto A. Algorithms for testing COVID-19 focused on use of RT-PCR and high-affinity serological testing: A consensus statement from a panel of Latin American experts. International Journal of Infectious Diseases. 2021;103:260-267. doi:10.1016/j.ijid.2020.11.173

41. Sethuraman N, Jeremiah SS, Ryo A. Interpreting Diagnostic Tests for SARS-CoV-2. JAMA. 2020;323(22):2249. doi:10.1001/jama.2020.8259

42. Mattioli IA, Hassan A, Oliveira ON, Crespilho FN. On the Challenges for the Diagnosis of SARS-CoV-2 Based on a Review of Current Methodologies. ACS Sens. December 2020. doi:10.1021/acssensors.0c01382

43. Li Y, Yao L, Li J, et al. Stability issues of RT-PCR testing of SARS-CoV-2 for hospitalized patients clinically diagnosed with COVID-19. Journal of Medical Virology. 2020;92(7):903-908. doi:https://doi.org/10.1002/jmv.25786

44. Pray IW. Performance of an Antigen-Based Test for Asymptomatic and Symptomatic SARS-CoV-2 Testing at Two University Campuses - Wisconsin, September-October 2020. MMWR Morb Mortal Wkly Rep. 2021;69. doi:10.15585/mmwr.mm695152a3

45. Cheng MP, Yansouni CP, Basta NE, et al. Serodiagnostics for Severe Acute Respiratory Syndrome-Related Coronavirus-2. Ann Intern Med. June 2020. doi:10.7326/M20-2854

46. Timing of surgery following SARS-CoV-2 infection: an international prospective cohort study. Anaesthesia. n/a(n/a). doi:https://doi.org/10.1111/anae.15458

47. Mills GH. Respiratory complications of anaesthesia. Anaesthesia. 2018;73(S1):25-33. doi:https://doi.org/10.1111/anae.14137

48. Kantor O, Wakeman M, Weiss A, et al. Axillary Management After Neoadjuvant Endocrine Therapy for Hormone Receptor-Positive Breast Cancer. Ann Surg Oncol. August 2020. doi:10.1245/s10434020-09073-6

49. Kantor O, Pesce C, Liederbach E, Wang C-H, Winchester DJ, Yao K. Are the ACOSOG Z0011 trial findings being applied to breast cancer patients undergoing neoadjuvant chemotherapy? The breast journal. 2017;23(5):554-562.

50. Giuliano AE, Ballman KV, McCall L, et al. Effect of Axillary Dissection vs No Axillary Dissection on 10-Year Overall Survival Among Women With Invasive Breast Cancer and Sentinel Node Metastasis: The ACOSOG Z0011 (Alliance) Randomized Clinical Trial. JAMA. 2017;318(10):918-926. doi:10.1001/jama.2017.11470

51. Moo T-A, Edelweiss M, Hajiyeva S, et al. Is Low-Volume Disease in the Sentinel Node After Neoadjuvant Chemotherapy an Indication for Axillary Dissection? Ann Surg Oncol. 2018;25(6):1488-1494. doi:10.1245/s10434-018-6429-2

52. Moo T-A, Pawloski KR, Flynn J, et al. Is Residual Nodal Disease at Axillary Dissection Associated with Tumor Subtype in Patients with Low Volume Sentinel Node Metastasis After Neoadjuvant Chemotherapy? Ann Surg Oncol. April 2021. doi:10.1245/s10434-021-09910-2

53. Comparison of Axillary Lymph Node Dissection With Axillary Radiation for Patients With Node-Positive Breast Cancer Treated With Chemotherapy. 2021. https://clinicaltrials.gov/ct2/show/NCT01901094. 
54.

Almahariq MF, Levitin R, Quinn TJ, et al. Omission of Axillary Lymph Node Dissection is Associated with Inferior Survival in Breast Cancer Patients with Residual N1 Nodal Disease Following Neoadjuvant Chemotherapy. Ann Surg Oncol. 2021;28(2):930-940. doi:10.1245/s10434-020-08928-2

55. Hammond JB, Parnall TH, Scott DW, et al. Gauging the efficacy of neoadjuvant endocrine therapy in breast cancer patients with known axillary disease. J Surg Oncol. June 2020. doi:10.1002/jso.26047

56. Bi Z, Liu J, Chen P, et al. Neoadjuvant chemotherapy and timing of sentinel lymph node biopsy in different molecular subtypes of breast cancer with clinically negative axilla. Breast Cancer. 2019;26(3):373377. doi:10.1007/s12282-018-00934-3

57. Tuttle TM, Burke EE. Bilateral Mastectomy: Doubling Down on Complications? Ann Surg Oncol. 2015;22(11):3407-3408. doi:10.1245/s10434-015-4629-6

58. Valero MG, Muhsen S, Moo T-A, et al. Increase in Utilization of Nipple-Sparing Mastectomy for Breast Cancer: Indications, Complications, and Oncologic Outcomes. Ann Surg Oncol. 2020;27(2):344-351. doi:10.1245/s10434-019-07948-x

59. Angarita FA, Acuna SA, Cordeiro E, et al. Thirty-day postoperative morbidity and mortality in elderly women with breast cancer: an analysis of the NSQIP database. Breast Cancer Res Treat. 2018;170(2):373-379. doi:10.1007/s10549-018-4747-5

60. O'Connell RL, Baker E, Trickey A, et al. Current practice and short-term outcomes of therapeutic mammaplasty in the international TeaM multicentre prospective cohort study. Br J Surg. 2018;105(13):17781792. doi:10.1002/bjs.10959

61. Cavalcante FP, Novita GG, Millen EC, Zerwes FP, Freitas Junior R. Breast reconstruction and coronavirus pandemic. J Plast Reconstr Aesthet Surg. 2021;74(3):644-710. doi:10.1016/j.bjps.2020.08.103

62. Desai A, Gainor JF, Hegde A, et al. COVID-19 vaccine guidance for patients with cancer participating in oncology clinical trials. Nature Reviews Clinical Oncology. March 2021:1-7. doi:10.1038/s41571021-00487-z

63. Corti C, Crimini E, Tarantino P, et al. Current Perspectives: SARS-CoV-2 vaccines for cancer patients: a call to action. European Journal of Cancer. February 2021:S0959804921000824. doi:10.1016/j.ejca.2021.01.046

64. Marra A, Generali DG, Zagami P, et al. LBA77 Anti-SARS-CoV-2 antibody response in patients with cancer and oncology healthcare workers: A multicenter, prospective study. Annals of Oncology. 2020;31:S1206. doi:10.1016/j.annonc.2020.08.2318

65. Miraglia JL, Abdala E, Hoff PM, et al. Immunogenicity and Reactogenicity of 2009 Influenza A (H1N1) Inactivated Monovalent Non-Adjuvanted Vaccine in Elderly and Immunocompromised Patients. PLOS ONE. 2011;6(11):e27214. doi:10.1371/journal.pone.0027214

66. Brydak LB, Guzy J, Starzyk J, Machała M, Góźdź SS. Humoral immune response after vaccination against influenza in patients with breast cancer. Support Care Cancer. 2001;9(1):65-68. doi:10.1007/s005200000186

67. Ward EM, Flowers CR, Gansler T, Omer SB, Bednarczyk RA. The importance of immunization in cancer prevention, treatment, and survivorship. CA: a cancer journal for clinicians. 2017;67(5):398-410.

68. Loulergue P, Alexandre J, Iurisci I, et al. Low immunogenicity of seasonal trivalent influenza vaccine among patients receiving docetaxel for a solid tumour: results of a prospective pilot study. British Journal of Cancer. 2011;104(11):1670-1674. doi:10.1038/bjc.2011.142

69. Grimm L, Destounis S, Dogan B, Nicholson B, Dontchos B, Sonnenblick E. SBI Recommendations for the Management of Axillary Adenopathy in Patients with Recent COVID-19 Vaccination. :3. 
70 .

Local Reactions, Systemic Reactions, Adverse Events, and Serious Adverse Events: Moderna COVID-19 Vaccine | CDC. https://www.cdc.gov/vaccines/covid-19/info-byproduct/moderna/reactogenicity.html. Published February 19, 2021. Accessed February 26, 2021.

71. Edmonds CE, Zuckerman SP, Conant EF. Management of Unilateral Axillary Lymphadenopathy Detected on Breast MRI in the Era of Coronavirus Disease (COVID-19) Vaccination. American Journal of Roentgenology. February 2021:AJR.21.25604. doi:10.2214/AJR.21.25604

72. Thakkar A, Pradhan K, Jindal S, et al. Patterns of seroconversion for SARS-CoV-2 IgG in patients with malignant disease and association with anticancer therapy. Nature Cancer. March 2021:1-8. doi:10.1038/s43018-021-00191-y 